\title{
In situ attosecond pulse characterization techniques to measure the electromagnetic phase
}

\author{
M. Spanner* \\ National Research Council of Canada, 100 Sussex Drive, Ottawa, ON K1A OR6, Canada \\ J. B. Bertrand \\ Center for Optics, Photonics and Lasers (COPL), Université Laval, Québec G1V 0A6, Canada \\ D. M. Villeneuve \\ Joint Attosecond Science Laboratory, National Research Council of Canada and University of Ottawa, 100 Sussex Drive, Ottawa, \\ ON K1A 0R6, Canada
}

(Received 31 May 2016; published 12 August 2016)

\begin{abstract}
A number of techniques have been developed to characterize the attosecond emission from high-order-harmonic sources. These techniques are broadly classified as ex situ, where the attosecond pulse train photoionizes a target gas in the presence of an infrared field, and in situ, where the measurement takes place in the medium in which the attosecond pulses are generated. It is accepted that ex situ techniques measure the characteristics of the electromagnetic field, including the phase of the recombination transition moment of the emitting atom or molecule, when the phase of the second medium is known. However, there is debate about whether in situ techniques measure the electromagnetic field, or only the characteristics of the recolliding electron before recombination occurs. We show numerically that in situ measurements are not sensitive to the recombination phase, when implemented in the perturbative regime as originally envisioned, and that they do not measure the electromagnetic phase of the emission.
\end{abstract}

DOI: 10.1103/PhysRevA.94.023825

\section{INTRODUCTION}

Attosecond pulses are produced by the process of highorder-harmonic generation (HHG), usually in gases [1-3], but recently also in solids $[4,5]$. Because these pulses have an optical frequency that lies in the extreme ultraviolet (XUV) or soft $\mathrm{x}$-ray spectrum $(10-1000 \mathrm{eV})$, they are challenging to characterize. For example, techniques that are used to characterize short pulses in the visible and near infrared cannot be used because optical components such as beam splitters and second-harmonic crystals will not work in the XUV.

A variety of techniques have been developed to characterize attosecond pulses. Nonlinear (two-photon) techniques require high intensity attosecond pulses that are currently achieved only in a few laboratories [6,7]. One advantage of the XUV spectral character is that atoms can be photoionized. Therefore most techniques rely on using the attosecond pulses to photoionize target atoms. The observable is the electron kinetic energy spectrum emitted by the atoms, usually in the presence of an infrared laser field to dress the ionization process. In this paper, we refer to these techniques as ex situ, because the measurement takes place in a second medium. There is a second class of measurements in which the highorder-harmonic process is weakly perturbed by a dressing field in the generating medium itself. The spectrum of the high-order-harmonic spectrum emitted from the generating medium is then used to characterize the pulses. We refer to this second class of techniques as in situ, because the measurement takes place directly in the generating medium. Reference [8] reviews these two approaches and contains a number

\footnotetext{
*Corresponding author: michael.spanner@nrc.ca
}

of references to various attosecond pulse characterization techniques.

Attosecond pulses are usually produced as a train of pulses, but with some effort can be produced as a single isolated attosecond pulse [9]. Here we will only discuss measurements of attosecond pulse trains. Because an attosecond pulse is emitted during every half-optical cycle of the driving laser, the spectrum must be composed of integer harmonics of the driving laser frequency, separated by twice the laser frequency. Because of the alternating left-right symmetry of the emission, the emitted spectrum must be composed of odd integer harmonics. By measuring both the amplitude $A_{q}$ and phase $\phi_{q}$ of each of these harmonic orders $q$, one can reconstruct the attosecond pulse train as $E(t)=\Sigma_{q} A_{q} \exp \left(i q \omega_{0} t+i \phi_{q}\right)$, where $q$ are odd integers and $\omega_{0}$ is the driving laser frequency [10]. It is possible to measure the harmonic phase $\phi_{q}$ of a target atom or molecule by interfering the emission with that of a reference atom, the so-called mixed-gas approach [11-13].

Although in situ techniques are sometimes thought to be equivalent to ex situ ones, we will show that that is not true, at least for certain in situ techniques. The difference between the two methods lies in the inclusion of the transition dipole matrix element of the atom or molecule that generates the HHG. Measuring the recombination phase is important in, e.g., reconstructing molecular orbitals [14]. According to the three-step model [15] of HHG, an electron is removed from the atom by the intense driving laser field. When the field changes direction in the next half-optical cycle, the electron is driven back to the atom, and it recombines. In doing so, it emits a photon whose energy is the sum of the kinetic energy of the electron plus the ionization potential of the atom. The phase of the emission is composed of individual phases from all three steps, the most important of which are the phase accumulated by the free electron over its trajectory, and the 
recombination phase. The recombination phase is associated with the photoionization phase of the same atom or molecule through time reversal arguments. The clearest example of photoionization phase jumps are those associated with Cooper minima in atoms; for example, the Cooper minimum seen in argon [16].

We will show that in situ techniques that use a weak perturbing field are not sensitive to the recombination phase of the atom, such as the Cooper minimum phase jump, whereas ex situ techniques are sensitive to the recombination phase. Thus in situ techniques can be used to characterize attosecond pulse trains, but only up to the recombination phase.

We use the word "perturbative" to refer to the effect of adding a weak second-harmonic field to the strong fundamental pulse. The process of HHG itself is highly nonperturbative. It has been shown that a photon picture can be used to describe the effect of a weak second-harmonic field on the HHG process [17]. It is this perturbative model that is needed to interpret in situ measurements.

\section{EX SITU TECHNIQUES}

The first measurements of attosecond pulses were done using ex situ techniques. The first such technique [10,18] was called RABBIT (reconstruction of attosecond beating by interference of two-photon transitions, also referred to as RABITT). In the RABBIT technique, the attosecond pulse train is focused into a second gas jet containing a reference atom such as neon. Each attosecond pulse creates a burst of photoelectrons, and due to the periodicity of the pulse train, the electron kinetic energy distribution is concentrated around peaks associated with the discrete high-order-harmonic spectrum, i.e., odd harmonics. A relatively weak infrared field $\left(\approx 10^{12} \mathrm{~W} / \mathrm{cm}^{2}\right)$, derived from the laser pulse that generates the high-order-harmonic emission in the first gas jet, is focused together with the attosecond pulse train in the second gas jet. The infrared field serves to couple together adjacent photoelectron energy peaks. After photoionization, the infrared field causes continuum-continuum transitions, resulting in the formation of sidebands. The sidebands can be produced through two different quantum paths, either absorbing or emitting one infrared photon, i.e., $\omega_{q}=(q+1) \omega_{0}-\omega_{0}$, or $\omega_{q}=(q-1) \omega_{0}+\omega_{0}$, where $q$ is an even integer and $\omega_{0}$ is the infrared laser central frequency.

Due to interference between the two quantum paths, the amplitude of the sidebands oscillate as the time delay $\tau$ between the attosecond pulse train and the laser pulse varies. The sideband intensity $S_{q} \propto \sin \left(2 \omega_{0} \tau+\phi_{q-1}-\phi_{q+1}+\phi_{q-1}^{\text {atom }}-\right.$ $\phi_{q+1}^{\text {atom }}$, where $\phi$ are the harmonic phases, and $\phi^{\text {atom }}$ is the photoionization phase of the target atom. Assuming that the photoionization phase is known or is flat, this provides a measure of the harmonic phases $\phi_{q}$.

Since the introduction of RABBIT, a variety of similar techniques have been developed [19-23] to characterize both attosecond pulse trains and isolated attosecond pulses. All of these techniques rely on photoionizing a target atom with the attosecond pulses.

\section{IN SITU TECHNIQUES}

The in situ class of attosecond pulse measurements is distinguished from ex situ techniques, in that the measurement takes place in the same medium that is used to generate the attosecond pulses. This approach is somewhat simpler experimentally than ex situ techniques because no photoelectron spectrometer is needed. The first such technique was introduced by Dudovitch et al. [24]. Here, a second laser pulse at the second harmonic of the generating infrared laser pulse is focused collinearly with the generating pulse into the generating medium. The $2 \omega_{0}$ pulse has an intensity that is typically less than $1 \%$ of the main pulse. The weak perturbing pulse breaks the left-right symmetry of the highorder-harmonic generation process, leading to the emission of even harmonics of the infrared laser frequency. Just as with the RABBIT technique, the delay $\tau$ between the two pulses causes a modulation in the intensity of the even harmonic order sidebands. This modulation then determines the harmonic phase $\phi_{q}$.

A variety of related in situ techniques have been developed. Using a perpendicular $2 \omega_{0}$ field, both the ionization and recombination times of each harmonic can be measured [25]. Using a noncollinear $2 \omega_{0}$ pulse, an isolated attosecond pulse was characterized both in time and in space [26].

Here we briefly describe the original collinear in situ technique [24]. The strong field approximation (SFA) is commonly used to calculate HHG emission [27]. The expression for the time-dependent dipole $\boldsymbol{d}(t)$ is

$$
\begin{aligned}
\boldsymbol{d}_{1}(t)= & -i \int d \boldsymbol{k} \int_{0}^{t} d t^{\prime}\left\langle\psi_{0}|\boldsymbol{r}| \boldsymbol{k}+\boldsymbol{A}(t)\right\rangle \\
& \times e^{-i S_{1}} e^{-I_{p}\left(t-t^{\prime}\right)} \boldsymbol{E}\left(t^{\prime}\right) \cdot\left\langle\boldsymbol{k}+\boldsymbol{A}\left(t^{\prime}\right)|\boldsymbol{r}| \psi_{0}\right\rangle .
\end{aligned}
$$

Here $\psi_{0}$ is the one-electron wave function describing the ground state, $\boldsymbol{k}$ is the canonical momentum, $\boldsymbol{A}$ is the vector potential of the laser field, $S_{1}=\int_{t^{\prime}}^{t} d t^{\prime \prime}\left[\boldsymbol{k}+\boldsymbol{A}\left(t^{\prime \prime}\right)\right]^{2} / 2$ is the classical action of the continuum electron, and $I_{p}$ is the ionization potential. Time $t$ represents the time of emission, and $t^{\prime}$ represents the time of ionization. The classical action $S_{1}$ is the integral of the kinetic energy of the continuum electron.

Dudovich et al. [24] showed that a weak second harmonic field with vector potential $A_{2} \sin \left(2 \omega_{0} t+\phi\right)$ has the effect of changing the classical action

$$
\begin{gathered}
S_{2}(t, \phi)=S_{1}(t)-\sigma(t, \phi), \\
\sigma(t, \phi)=\int_{t^{\prime}(t)}^{t} d \tau v\left(\tau, t^{\prime}\right) A_{2}(\tau, \phi) .
\end{gathered}
$$

Here $v$ is the velocity of the unperturbed electron trajectory. The additional phase $\sigma$ is added to the electron trajectory for one half-optical cycle, and is subtracted on the next half-optical cycle. This phase difference causes a slight difference in the emission phase for positive and negative half-cycles, causing a symmetry breaking and leading to the emission of even harmonics.

The analysis in Dudovich et al. [24] showed that the optical phase difference $\phi_{q}^{\max }$ which maximized the even harmonic order $q$ gives a measure of the emission time of that harmonic, and that the effect of the Coulomb potential was negligible. 
We note here that, in the limit of a weak perturbing field amplitude, the second harmonic field only enters into the expression for $S_{2}$ which replaces $S_{1}$ in Eq. (1). It does not change the recombination dipole moment. (If the second harmonic field is increased in intensity such that it is no longer a weak perturbation, then higher order corrections to the SFA model are introduced, and each of the three steps of the HHG process can be affected.) Our analysis suggests that in situ measurements are not sensitive to the recombination dipole moment phase. We confirm this conjecture later in this paper.

\section{TIME-DEPENDENT SCHRÖDINGER EQUATION SIMULATIONS}

Here we perform numerical experiments to investigate both approaches to attosecond pulse characterization. We numerically calculate the emission from a model atom that has a phase jump engineered into its recombination dipole matrix element. We can use the attosecond pulse train emitted from the model atom to photoionize a second atom with a flat phase, and record the photoelectron kinetic energy spectrum in the presence of a weak infrared field, just as the RABBIT technique. Alternately, we can perturb the highorder-harmonic generation process with a parallel polarized $2 \omega_{0}$ field in an analog to the original in situ technique, and see whether the phase jump is present in the measurement.

We numerically solve the single-electron one-dimensional time-dependent Schrödinger equation (TDSE) in the dipole approximation

$$
i \frac{\partial}{\partial t} \Psi(x, t)=\left[-\frac{1}{2} \frac{\partial^{2}}{\partial x^{2}}+V(x)+E(t) \cdot x\right] \Psi(x, t),
$$

where $V(x)$ is the core potential and $E(t)$ is the electric field of the laser. Hartree atomic units $\left(\hbar=m_{e}=e=1\right)$ are used for all equations. The TDSE was solved using the standard Fourier split-operator method. The HHG spectrum $W_{\mathrm{HHG}}(\omega)$ is computed from the Fourier transform of the dipole acceleration

$$
W_{\mathrm{HHG}}(\omega)=\int d t \ddot{d}(t) e^{i \omega t}
$$

where $\ddot{d}(t)$ is calculated using Ehrenfest's theorem [28]

$$
\ddot{d}(t)=-\left\langle\Psi(t)\left|\frac{\partial V(x)}{\partial x}\right| \Psi(t)\right\rangle .
$$

When simulating the RABBIT technique, we first compute the HHG emission $E_{\mathrm{HHG}}(t) \equiv \ddot{d}(t)$, and then use $E_{\mathrm{HHG}}(t)$ as an input electric field for a second simulation that models the RABBIT measurement. The specific procedure for this RABBIT simulation is elaborated further below.

HHG from two model soft-core systems is considered, as shown in Fig. 1. The first is an atomiclike potential $\left(V_{1}\right)$ which does not exhibit a phase jump in the HHG spectrum, and the second is a two-center potential $\left(V_{2}\right)$ which does show a phase jump. These two potentials are defined as

$$
V_{1}(x)=-\frac{1}{\sqrt{x^{2}+a_{1}^{2}}}
$$
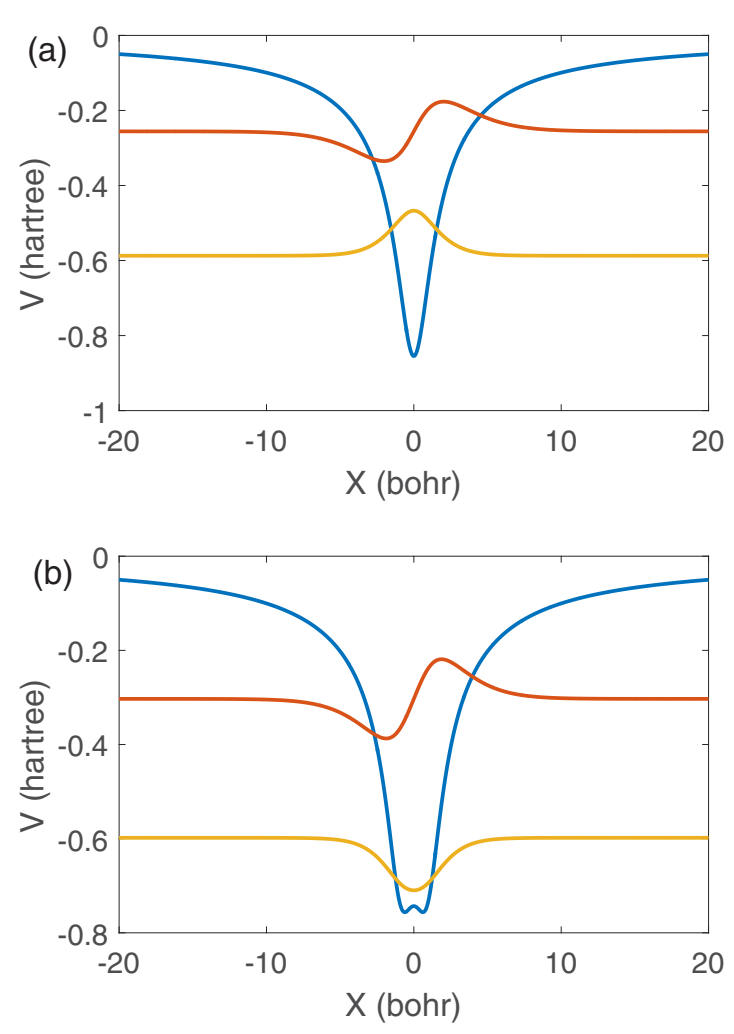

FIG. 1. Model potentials used in calculations. The TDSE calculations used two slightly different potentials. (a) is the single-well potential $V_{1}$, and (b) is the double-well potential $V_{2}$. The double-well potential exhibits a phase jump in the recombination dipole moment. Also shown are the two lowest eigenstates of the potentials, with their vertical positions representing the energy of the eigenstate.

and

$$
V_{2}(x)=-\frac{0.5}{\sqrt{(x+R / 2)^{2}+a_{2}^{2}}}-\frac{0.5}{\sqrt{(x-R / 2)^{2}+a_{2}^{2}}},
$$

where $a_{1}=1.17, a_{2}=1$, and $R=1.8$ a.u. In the following, we consider the HHG from the ground state of both systems, which were found by propagating in complex time. The ionization potentials for the two systems are $16.0 \mathrm{eV}$ (for atomic) and $16.2 \mathrm{eV}$ (for two-center).

In order to generate a clear and sharp phase jump in the harmonic emission, we remove the first excited state from the propagation of the two-center system. This has the effect of drastically reducing both the polarizability and the field-induced distortions of the initial state. Formally, this is accomplished by using the modified Hamiltonian $H \rightarrow$ $\widehat{P}_{1} H \widehat{P}_{1}$, where $\widehat{P}_{1}=1-\left|\varphi_{1}\right\rangle\left\langle\varphi_{1}\right|$ is the projection operator that projects out the first excited state $\left|\varphi_{1}\right\rangle$ (see Fig. 1). In practice, this modified Hamiltonian is implemented by propagating with the original Hamiltonian, but then applying $\widehat{P}_{1}$ to the wave function after each step and renormalizing. To separate the long and short trajectory contributions to the HHG emission, which is also essential for generating a clean phase jump in the two-center model, a complex absorbing boundary is used in the simulations to remove the part of the continuum wave packet that corresponds to the long trajectories. The parameters of the absorbing boundary were 

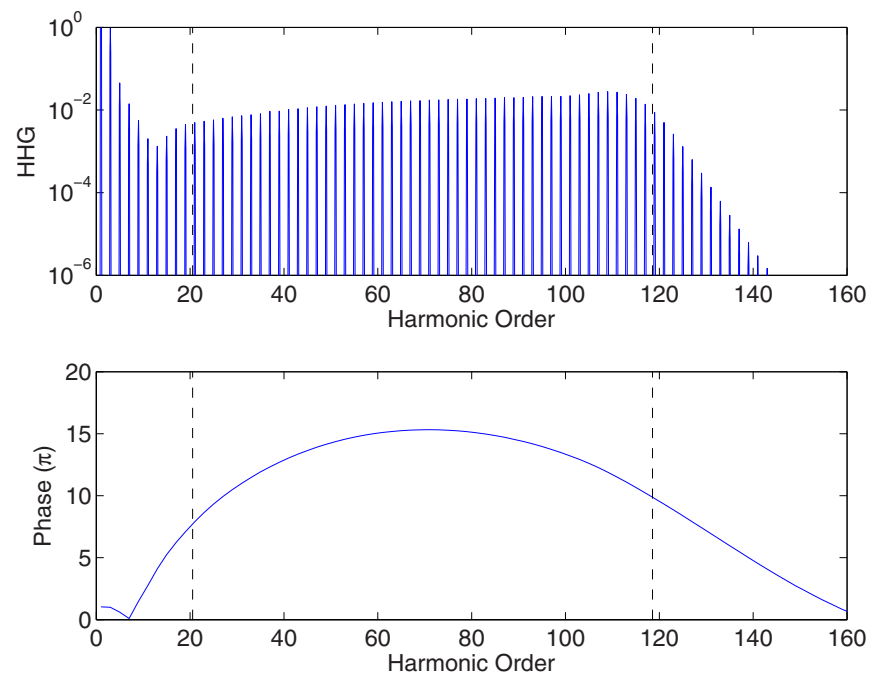

FIG. 2. High-order-harmonic emission from the atomiclike potential $\boldsymbol{V}_{1}$. The one-electron time-dependent Schrödinger equation is solved for a single-center soft-core Coulomb potential in the presence of an intense 1600-nm laser field. The intensity (top) and phase (bottom) of the emitted high-order-harmonic emission are shown. The horizontal axis is the emitted photon energy in units of the 1600-nm photon frequency.

tuned by monitoring a window Fourier transform of $\ddot{d}(t)$. For the intensity used in the results presented below, we found that an absorbing width of 26 a.u. that was turned on at position $x=54$ a.u. was adequate when using the absorbing boundary outlined in [29]. The resulting harmonic spectra plotted below then contain only contributions corresponding to the short trajectories.
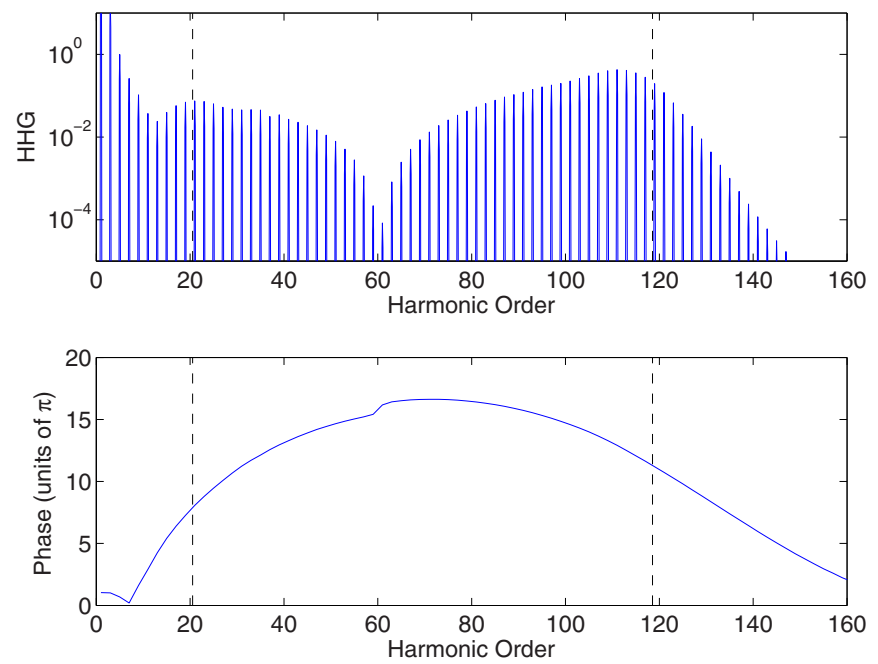

FIG. 3. High-order-harmonic emission from the two-center potential $\boldsymbol{V}_{2}$. The one-electron time-dependent Schrödinger equation is solved for a two-center Coulomb potential in the presence of an intense 1600-nm laser field. The intensity (top) and phase (bottom) of the emitted high-order-harmonic emission are shown. A minimum in intensity is seen around harmonic order 60 . A corresponding $\pi$ phase jump is seen in the bottom plot.
The harmonic spectrum generated from these model systems is shown in Figs. 2 and 3. These HHG spectra were generated by applying a ten-cycle flattop pulse of $1600 \mathrm{~nm}$ $\left(\omega_{0}=0.775 \mathrm{eV}=0.0285\right.$ a.u.) radiation defined as $E(t)=$ $\mathcal{E}_{0} \sin \left(\omega_{0} t\right)$. The field strength $\mathcal{E}_{0}=0.0535$ a.u. $\left(10^{14} \mathrm{~W} / \mathrm{cm}^{2}\right)$ was used. For reference, the vertical dashed lines in Figs. 2 and 3 denote the threshold harmonic order $I_{p} / \omega_{0} \approx 21$ and the cut-off harmonic $\left(3.17 U_{p}+I_{p}\right) / \omega_{0} \approx 119$, where $U_{p}=$ $E^{2} /\left(4 \omega_{0}^{2}\right)$ is the ponderomotive potential. The atomic system shows a smooth amplitude and phase structure across the harmonic plateau, while the two-center system exhibits a clear minimum in the amplitude at harmonic order 61 with a corresponding $\pi$ phase jump at the same harmonic. Within the three-step model [15], the phase jump seen in Fig. 3 originates from the recombination step.

\section{A. Modeling the in situ technique}

In order to model the in situ measurement, we add a second harmonic field with $1 \%$ electric field amplitude,

$$
E(t)=\mathcal{E}_{0}\left[\sin \left(\omega_{0} t\right)+0.01 \sin \left(2 \omega_{0} t+\varphi_{2}\right)\right],
$$

where $\varphi_{2}$ is the relative phase between the two colors. An example HHG spectrum for the two-center potential using this two-color field with $\varphi_{2}=0$ is shown in Fig. 4. As discussed above, the addition of the second harmonic breaks the spatial symmetry of the problem and leads to the emission of evenorder harmonics that can be seen in Fig. 4. Following the prescription for the in situ measurement [24], the HHG spectra were calculated for all phases between $\varphi_{2}=0$ and $\pi$. Figure 5 plots the amplitudes of all the even harmonics (integrated over each individual spectral peak) as a function of $\varphi_{2}$, for both model systems. These amplitudes have been normalized to the peak value as a function of $\varphi_{2}$ for each even order. The white circles in Fig. 5 track the position of the maximum of each even order as a function of $\varphi_{2}$. In the in situ method [24], the position of the peaks (the curve traced out by connecting the white circles) is used to retrieve phase and timing information related to the emission of each harmonic. From Fig. 5, it is seen that the even harmonics for both the atomic and two-center potentials are essentially identical. Importantly, there is no hint of any differences near harmonic order 60 where one might expect to see the phase jump present in the harmonics for the

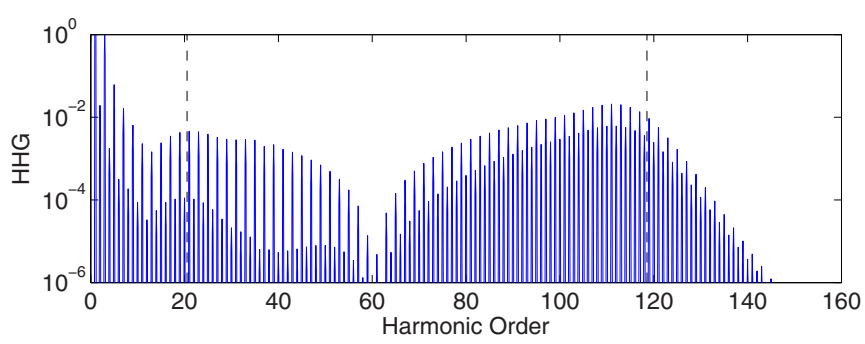

FIG. 4. Example of the in situ high-order-harmonic spectrum. The one-electron time-dependent Schrödinger equation is solved for the two-center potential in the presence of an intense 1600-nm laser field. A weak 800 -nm dressing field is used to break the left-right symmetry of the HHG process, leading to emission of even harmonic orders. 

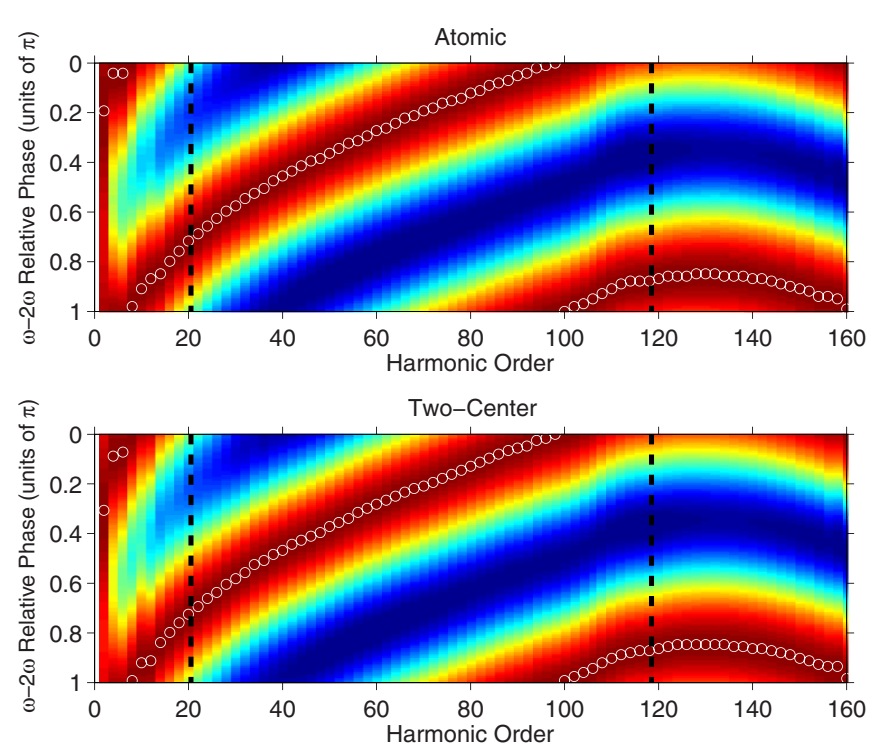

FIG. 5. In situ even-order harmonics. The intensities of the even harmonic orders are taken from Fig. 4 as a function of the relative phase $\varphi_{2}$ between the 1600- and 800-nm laser fields (vertical axis). The color scale indicates the intensity ratio. The white circles show the positions at which the even harmonic intensity is maximized. By comparing the emission from a single-well potential (top) with that from a two-center potential (bottom), it can be seen that the in situ measurement does not show a phase jump at H60.

two-center system. These results demonstrate that the in situ measurement is not sensitive to the phase of the recombination step.

\section{B. Modeling the $e x$ situ technique}

To model the ex situ (or RABBIT) technique, the HHG radiation from Figs. 2 and 3, which was generated by the single-color driving field, is used as an input field to a second simulation that now computes photoelectron spectra. For the ionization target used to model the RABBIT measurement we use a soft-core single-center potential with the soft-core parameter of $a_{1}=1.59$ that corresponds to an $I_{p}$ of $12.3 \mathrm{eV}$. In addition to the HHG radiation, the RABBIT method also uses a weak IR field to streak the photoelectrons ejected by the HHG radiation, which generates sidebands on the photoelectron peaks that interfere to reveal the relative phase of the different harmonics present in the HHG radiation.

In order to generate a clean photoelectron spectrum, some preconditioning of the $\mathrm{HHG}$ radiation was applied before using in the RABBIT simulations. A Hann window function is first applied to the $\mathrm{HHG}$ radiation $E_{\mathrm{HHG}}(t) \equiv \ddot{d}(t)$ computed above:

$$
E_{\mathrm{HHG}}(t) \rightarrow \sin ^{2}\left(\frac{2 \pi t}{T_{\max }}\right) E_{\mathrm{HHG}}(t),
$$

where $T_{\max }$ is the total time range of the simulation that computed $E_{\mathrm{HHG}}(t)$, which was ten cycles of the $1600-n m$ driving field. Applying the Hann window cleans up any possible edge-related artifacts and discontinuities that might be present in the numerically computed harmonic radiation $E_{\mathrm{HHG}}(t)$. Next, $E_{\mathrm{HHG}}(t)$ is put through a high-pass Fourier filter that removes the sub- $I_{p}$ harmonics below harmonic

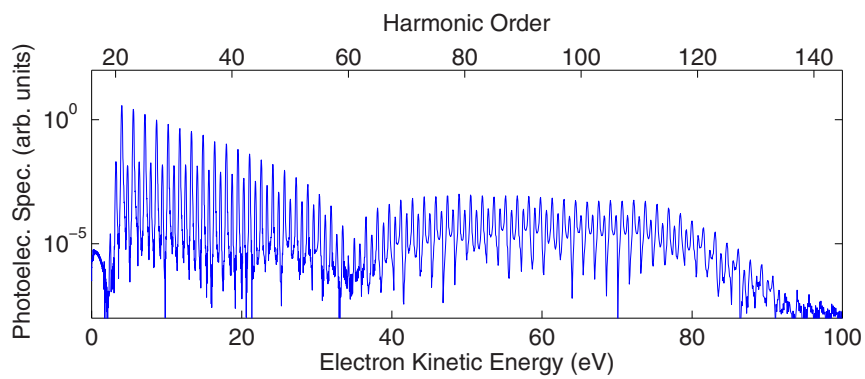

FIG. 6. Example of the photoelectron kinetic energy spectrum from a RABBIT measurement. The electromagnetic field emitted from the two-center potential (Fig. 3) is used to photoionize a target gas. A weak 1600-nm laser field is used to dress the photoionization process for the RABBIT measurement, producing sidebands on the photoelectron peaks. The resulting continuum photoelectron spectrum $\left[\left|\Psi\left(p^{2} / 2\right)\right|^{2}\right]$ is plotted as a function of kinetic energy.

order 20, which become increasingly intense for lower frequencies. This high-pass filtering removes the possibility that the intense sub- $I_{p}$ harmonics can streak the photoelectrons generated by single-photon ionization from the plateau region, and also ensures that we avoid accessing possible bound state resonances within the photoionization target atom. It should be noted that this preconditioning and filtering procedure does not alter the relative phases across the plateau region of the HHG spectrum.

As required by the RABBIT method, the (filtered) harmonic radiation is added to weak radiation at the fundamental frequency to give the mixed field

$$
E(t)=E_{\mathrm{HHG}}(t)+\mathcal{E}_{w} \sin \left(\omega_{0} t+\varphi_{R}\right),
$$

where the weak fundamental had the intensity of $1.4 \times 10^{9} \mathrm{~W} / \mathrm{cm}^{2}\left(\mathcal{E}_{w}=0.0002\right.$ a.u. $)$, and the phase $\varphi_{R}$ controls the relative timing between the fundamental and the harmonic radiation $E_{\mathrm{HHG}}(t)$. The Schrödinger equation, Eq. (4), is numerically solved with this $E(t)$ for a time range of ten cycles of the fundamental. In order to ensure that our computational grid is large enough to accommodate the full continuum wave function as is spreads away from the core, we use a spatial grid with a range of 5120 a.u. and a total number of points $2^{15}$. The resulting continuum wave function $\Psi_{c}(x)$ is extracted by taking the final wave function and projecting out the ground and first excited states. The photoelectron energy spectrum is than computed by Fourier transforming the wave function from space to momentum, $\Psi(p)=\mathcal{T}_{F}[\Psi(x)]$, and plotting against the kinetic energy $p^{2} / 2$.

An example photoelectron spectrum is shown in Fig. 6 using the HHG emission from the two-center potential $V_{2}$ and for a RABBIT phase of $\varphi_{R}=0$. Also shown in Fig. 6 is the mapping of kinetic energy to the corresponding harmonic order $\left(O_{H}\right)$ using the relation $O_{H}=\left(p^{2} / 2-I_{p}\right) / \omega_{0}$, where $I_{p}$ is ionization potential of the target atom used in the RABBIT measurement. Figure 7 plots the integrated amplitudes of the photoelectron peaks corresponding to the even harmonic orders as a function of the RABBIT phase $\varphi_{R}$. The white circles track the maxima of the even-order peaks, as was done in Fig. 5. It can be seen that there is a sharp jump in the position of the maxima around harmonic order 60 for the 

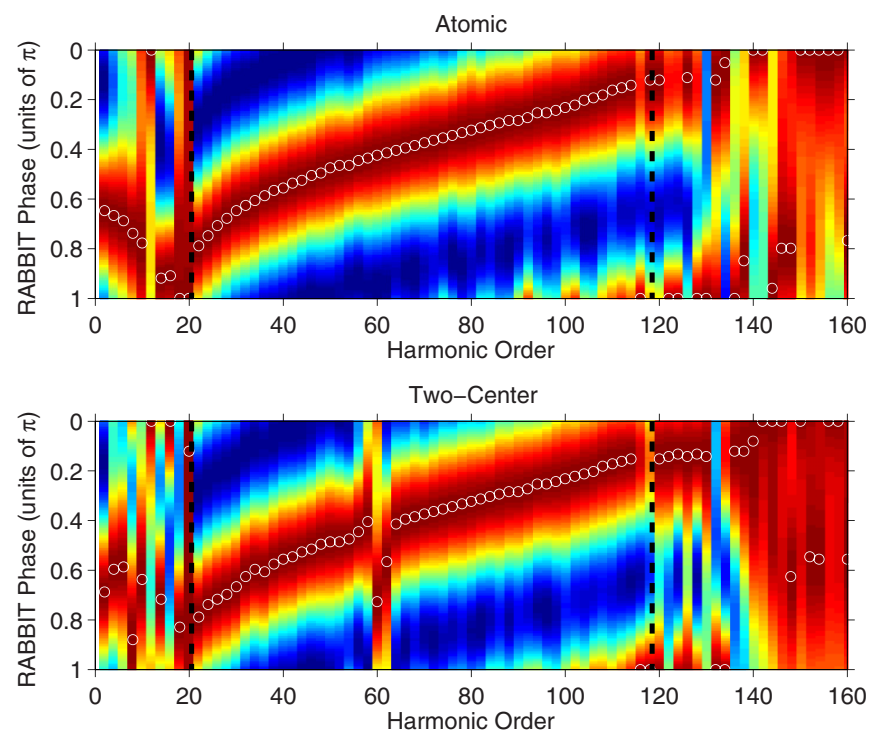

FIG. 7. Phase determined from RABBIT measurement. The integrated amplitudes of the photoelectron peaks corresponding to even-order harmonics, plotted as the color scale, versus the phase delay $\varphi_{R}$ of the dressing 1600-nm field relative to the attosecond pulse train. The white circles show the positions at which the sideband intensity is maximized. A clear phase shift is seen at harmonic order 60 , corresponding to the $\pi$ phase jump in the electromagnetic phase.

RABBIT simulations of the two-center potential that is not present in the RABBIT simulation of the atomiclike potential. This jump signals the presence of the $\pi$ phase jump seen in the HHG spectra of the two-center seen in Fig. 3, and demonstrates that, unlike the case for the in situ method, the RABBIT method is indeed sensitive to the phase structures present in the recombination step of the HHG process.

\section{CONCLUSION}

We have shown that the in situ attosecond pulse characterization technique, in which a copolarized second harmonic of the driving laser is used to perturb the high-order-harmonic process in the generating medium, is not sensitive to the atomic recombination phase, when the $2 \omega_{0}$ field is weak enough such that only the lowest order perturbation is active. On the other hand, ex situ techniques such as RABBIT include the atomic phase twice - the recombination phase of the generating atom and the photoionization phase of the detecting atom. This finding likely also applies to the noncollinear in situ version $[26,30]$, although we have not explicitly demonstrated it in this paper.

There are situations in which in situ measurements do not need to be sensitive to the recombination phase, such as when one wishes to characterize the motion of the electron. In those cases, it is desirable not to include the recombination phase. Examples of this class of measurements are where the time of ionization and recombination are measured [25], or when the band structure of a solid is probed [5].

Note that the phase jump in Fig. 7 is only visible in two harmonic orders around H60. Because this measurement gives the derivative of the spectral phase, $\left(\phi_{q+1}-\phi_{q-1}\right) / 2 \omega_{0}$, the phases above and below the phase jump are unchanged. Such a phase jump is usually associated with a minimum in the harmonic emission. Therefore it can be experimentally difficult to identify such a sharp phase jump.
[1] P. B. Corkum and F. Krausz, Nat. Phys. 3, 381 (2007).

[2] A. Scrinzi, M. Y. Ivanov, R. Kienberger, and D. M. Villeneuve, J. Phys. B: At., Mol. Opt. Phys. 39, R1 (2006).

[3] F. Krausz and M. Y. Ivanov, Rev. Mod. Phys. 81, 163 (2009).

[4] S. Ghimire, A. D. DiChiara, E. Sistrunk, P. Agostini, L. F. DiMauro, and D. A. Reis, Nat. Phys. 7, 138 (2011).

[5] G. Vampa, T. J. Hammond, N. Thiré, B. E. Schmidt, F. Légaré, C. R. McDonald, T. Brabec, and P. B. Corkum, Nature (London) 522, 462 (2015).

[6] J. E. Kruse, P. Tzallas, E. Skantzakis, C. Kalpouzos, G. D. Tsakiris, and D. Charalambidis, Phys. Rev. A 82, 021402 (2010).

[7] E. J. Takahashi, P. Lan, O. D. Mücke, Y. Nabekawa, and K. Midorikawa, Nat. Commun. 4, 2691 (2013).

[8] K. T. Kim, D. M. Villeneuve, and P. B. Corkum, Nat. Photonics 8, 187 (2014).

[9] G. Sansone, E. Benedetti, F. Calegari, C. Vozzi, L. Avaldi, R. Flammini, L. Poletto, P. Villoresi, C. Altucci, R. Velotta et al., Science 314, 443 (2006).

[10] Y. Mairesse, A. de Bohan, L. J. Frasinski, H. Merdji, L. C. Dinu, P. Monchicourt, P. Breger, M. Kovačev, R. Täeb, B. Carré et al., Science 302, 1540 (2003).
[11] J. B. Bertrand, H. J. Wörner, P. Salières, D. M. Villeneuve, and P. B. Corkum, Nat. Phys. 9, 174 (2013).

[12] B. K. McFarland, J. P. Farrell, P. H. Bucksbaum, and M. Guhr, Phys. Rev. A 80, 033412 (2009).

[13] N. Wagner, X. Zhou, R. Lock, W. Li, A. Wüest, M. Murnane, and H. Kapteyn, Phys. Rev. A 76, 061403 (2007).

[14] S. Haessler, J. Caillat, W. Boutu, C. Giovanetti-Teixeira, T. Ruchon, T. Auguste, Z. Diveki, P. Breger, A. Maquet, B. Carré et al., Nat. Phys. 6, 200 (2010).

[15] P. B. Corkum, Phys. Rev. Lett. 71, 1994 (1993).

[16] H. J. Wörner, H. Niikura, J. B. Bertrand, P. B. Corkum, and D. M. Villeneuve, Phys. Rev. Lett. 102, 103901 (2009).

[17] J. B. Bertrand, H. J. Wörner, H.-C. Bandulet, E. Bisson, M. Spanner, J.-C. Kieffer, D. M. Villeneuve, and P. B. Corkum, Phys. Rev. Lett. 106, 023001 (2011).

[18] P. M. Paul, E. S. Toma, P. Breger, G. Mullot, F. Augé, P. Balcou, H. G. Muller, and P. Agostini, Science 292, 1689 (2001).

[19] J. Itatani, F. Quéré, G. L. Yudin, M. Y. Ivanov, F. Krausz, and P. B. Corkum, Phys. Rev. Lett. 88, 173903 (2002).

[20] Y. Mairesse and F. Quéré, Phys. Rev. A 71, 011401 (2005).

[21] M. Kitzler, N. Milosevic, A. Scrinzi, F. Krausz, and T. Brabec, Phys. Rev. Lett. 88, 173904 (2002). 
[22] R. Lopez-Martens, K. Varjú, P. Johnsson, J. Mauritsson, Y. Mairesse, P. Salieres, M. B. Gaarde, K. J. Schafer, A. Persson, S. Svanberg et al., Phys. Rev. Lett. 94, 033001 (2005).

[23] M. Chini, S. Gilbertson, S. D. Khan, and Z. Chang, Opt. Express 18, 13006 (2010).

[24] N. Dudovich, O. Smirnova, J. Levesque, Y. Mairesse, M. Y. Ivanov, D. M. Villeneuve, and P. B. Corkum, Nat. Phys. 2, 781 (2006).

[25] D. Shafir, H. Soifer, B. D. Bruner, M. Dagan, Y. Mairesse, S. Patchkovskii, M. Y. Ivanov, O. Smirnova, and N. Dudovich, Nature (London) 485, 343 (2012).
[26] K. T. Kim, C. Zhang, A. D. Shiner, S. E. Kirkwood, E. Frumker, G. Gariepy, A. Naumov, D. M. Villeneuve, and P. B. Corkum, Nat. Phys. 9, 159 (2013).

[27] M. Lewenstein, Ph. Balcou, M. Yu. Ivanov, A. L'Huillier, and P. B. Corkum, Phys. Rev. A 49, 2117 (1994).

[28] K. Burnett, V. C. Reed, J. Cooper, and P. L. Knight, Phys. Rev. A 45, 3347 (1992).

[29] D. E. Manolopoulos, J. Chem. Phys. 117, 9552 (2002).

[30] K. T. Kim, C. Zhang, A. D. Shiner, B. E. Schmidt, F. Légaré, D. M. Villeneuve, and P. B. Corkum, Nat. Photonics 7, 958 (2013). 